\title{
Porcine acellular dermis-based breast reconstruction: complications and outcomes following adjuvant radiotherapy
}

\author{
Cho Ee Ng ${ }^{1}$ - Andrew Pieri ${ }^{1} \cdot$ Tarannum Fasih $^{1}$
}

Received: 22 February 2015 / Accepted: 1 July 2015 / Published online: 2 August 2015

(C) Springer-Verlag Berlin Heidelberg 2015

\begin{abstract}
Background Acellular dermal matrices (ADM) such as Strattice ${ }^{\mathrm{TM}}$ are increasingly used in UK during implantbased reconstruction. However, there are mixed opinions regarding the compatibility of radiotherapy treatment in pre- and post-reconstructed breasts. The aims of this study are to audit the rates of radiation induced complications in patients who underwent breast reconstruction using Strattice ${ }^{\mathrm{TM}}$ and establish whether there is an association between timing of radiotherapy and complication rates.

Methods Retrospective data collection was performed for all patients who underwent skin-sparing mastectomy and immediate or delayed Strattice ${ }^{\mathrm{TM}}$-based reconstruction, and received pre- or post-reconstruction radiotherapy from July 2010 to November 2014.

Results The age ranged from 33 to 78 years (mean age $51 \pm$ 10.6) with a mean follow-up time of 21 months. There were 25 Strattice $^{\mathrm{TM}}$-based reconstructions performed. Sixteen had delayed reconstruction, and 9 had skin-sparing mastectomy and immediate reconstruction. There were 4 (16\%) abandoned procedures due to inability to stretch the mastectomy flap secondary to poor skin compliance. Two women (8\%) presented 4 and 9 months later with wound breakdown. One case $(4 \%)$ developed severe capsular contracture following radiotherapy post-reconstruction and 1 case $(4 \%)$ of implant rupture. There were no episodes of extrusion or implant infection. Overall complication rates were $32 \%$. The majority (75\%) of complications occurred in breasts reconstructed
\end{abstract}

Cho Ee Ng

isao.nce@gmail.com

1 Surgical Department, Queen Elizabeth Hospital, Gateshead NE9 6SX, UK post-radiotherapy; however, this is not significant when analysed using chi-square $(p=0.43)$.

Conclusions Our evidence suggests that there is no difference in complication rates in pre- and post-radiation individuals; this would suggest that implant-based reconstruction using Strattice ${ }^{\mathrm{TM}}$ should not be an absolute contraindication in pre- or post-radiotherapy patients. However, when planning these procedures, it is paramount that the increased risks are emphasised to patients in order to better manage patient expectation in cases where complications arise.

Level of Evidence: Level III, risk / prognostic study.

Keywords Acellular dermal matrix $\cdot$ Strattice ${ }^{\mathrm{TM}} \cdot$ Breast reconstruction $\cdot$ Radiotherapy

\section{Introduction}

Increasingly, women are opting for breast reconstruction following breast cancer surgery. The number of implant-based reconstructions has risen to meet this demand [1]. Acellular dermal matrices (ADM) such as Strattice ${ }^{\mathrm{TM}}$ (LifeCell Corporation, Houston, Texas, USA) are increasingly used in the UK to reinforce the lower pole of the breast during implant-based breast reconstruction [2]. Radiotherapy is an important part of adjuvant treatment for breast cancer. Following radiotherapy treatment, the blood supply to the skin can become compromised [3-5]; as a consequence, there is an increased risk of tissue damage, infection, poor cosmetic outcome and even loss of the reconstructed breast in the post-radiotherapy population undergoing implant-based reconstruction using ADM [6-8]. Due to current evidence suggesting an increased risk of complications in women undergoing ADM-based reconstructions after completion of radiotherapy, many surgeons shun these as post-radiation reconstructive options. 
The aims of this study are to audit rates of radiation induced complications in patients who underwent breast reconstruction using Strattice ${ }^{\mathrm{TM}}$ and establish whether there is an association between timing of radiotherapy and complication rates.

\section{Patients and methods}

Retrospective data collection was performed for all patients who underwent immediate or delayed Strattice ${ }^{\mathrm{TM}}$-based reconstruction from July 2010 to November 2014 with a focus on those who received pre- or post-reconstruction radiation. Patients were offered different reconstructive options based on the size of their breast. Those with small to moderate size breast were offered Strattice ${ }^{\mathrm{TM}}$-based reconstruction. Patients with large, ptotic-shaped breast were offered reconstruction using dermal sling to reinforce the inframammary pole. Both groups were offered latissimus dorsi (LD)-based reconstruction as an alternative or reserve procedure. Ultimately, it was the patient's decision on which reconstruction they preferred.

There were two smokers within the group of patients undergoing Strattice-based reconstruction with radiotherapy. $\mathrm{Pa}-$ tients were divided into two groups. Group A delayed reconstruction (DR) (i.e., radiotherapy prior to reconstruction) and group B skin-sparing mastectomy (SSR) with immediate reconstruction (IR) (i.e., radiotherapy post-reconstruction). In group $\mathrm{A}$, there were two patients with previous wide local excision and radiotherapy. One of these had a recurrence of cancer and underwent mastectomy with immediate submuscular implant before a delayed Strattice ${ }^{\mathrm{TM}}$-based reconstruction. Another patient had bilateral breast reduction but then opted for mastectomy and Strattice ${ }^{\mathrm{TM}}$ reconstruction. There was also one patient that had to undergo a revision procedure for bottoming out due to a large expander.

Complications evaluated were those related to radiation exposure including failed procedure due to poor skin compliance with expansion, implant extrusion, capsular formation, wound infection and wound breakdown resulting in loss of implant.

In group $\mathrm{A}$, the procedure was delayed for a minimum of 1 year following radiotherapy. These patients underwent a basic clinical evaluation of their mastectomy flap, by performing a 'pinch test' and 'eye balling', assessing for skin elasticity, mobility and for obvious tissue damage due to radiation. Patients were also advised to gently massage the chest wall with body lotions on daily basis till surgery.

\section{Operative procedure}

A single dose of co-amoxiclav (or an alternative in cases of penicillin allergy) is administered at induction. Once removed from packaging, Strattice ${ }^{\mathrm{TM}}$ then undergoes a thorough 'washing' process by hand, in three separate bowls prior to implantation. This process takes approximately 20-30 min.
Following mastectomy, a subpectoral space is created by dissecting the lower border of the pectoralis major (PM) from the chest wall, as far as the sternum, medially, and up to the point of proposed cleavage, superiorly. The cavity is washed with warm saline to remove loose debris and fat. A $10 \times 16-\mathrm{cm}$ piece of Strattice ${ }^{\mathrm{TM}}$ is laid behind the lower mastectomy flap. The 1st suture is centred in the Strattice ${ }^{\mathrm{TM}}$ and the mid arc of the inframammary fold (IMF). The matrix is then anchored by way of three-point fixation through the Strattice ${ }^{\mathrm{TM}}$, the medial pole of PM and the medial limit of the IMF. Multiple interrupted sutures are then placed along the IMF as far, laterally, as the anterior axillary line. The cavity is measured and an appropriate expander is selected, matching the dimensions and placed in the subpectoral pouch created. Excess Strattice $^{\mathrm{TM}}$ is not trimmed but laid flat behind the pectoralis major in front of the expander without any folds. The PM is sutured to the matrix using inverted horizontal mattress sutures. Closure of the lateral space is performed to avoid migration of expander into the axilla. A single drain is placed, running along the IMF, between the skin and the matrix. A second drain is placed in the axilla for patients who underwent axillary clearance. A fully inflated expander was used for all skin-sparing mastectomies. In cases of delayed reconstruction, the amount of fluid in the expander was dependent on the subpectoral space. Patients are kept overnight for $24 \mathrm{~h}$ of intravenous antibiotics and discharged home with 1 week of oral antibiotics. The drain is removed when there is less than $30 \mathrm{ml}$ of output per $24 \mathrm{~h}$ for two consecutive days. If output remains persistent, the drain is removed at 3 weeks post-operatively. Patients are instructed to wear a support bra for 6 weeks. Initial follow-up occurs on post-operative day 5 for a wound check. This is performed by a breast specialist nurse. No community nurse was involved at any stage of the wound care.

Patients who have had a DR undergo inflation of the expander by a dedicated breast specialist nurse at least 5 weeks after surgery. The amount of fluid injected is variable and adjusted on an individual basis. In those having immediate reconstruction, following multidisciplinary review, the patients are referred to oncologists for further chemotherapy or radiotherapy treatment as required.

In those with DR, all patients underwent a second procedure to replace the expanders with implants after full expansion was achieved. The procedure was abandoned if skin became tight and shiny, and there was suspicion of failed inflation. Those with IR waited a further 6 months after radiation before an exchange procedure is performed.

\section{Results}

There were 64 Strattice ${ }^{\mathrm{TM}}$-based reconstructions identified that were performed from July 2010 to November 2014. 
Table 1 Complications

\begin{tabular}{ll}
\hline Complication & Number of cases, $N(\%)$ \\
\hline Poor skin compliance & $4(16)$ \\
Wound breakdown & $2(8)$ \\
Severe capsular contracture & $1(4)$ \\
Implant rupture & $1(4)$ \\
Total & $8(32)$ \\
\hline
\end{tabular}

Those who chose this preferred a less extensive surgery first rather than the more extensive LD reconstruction. The age ranged from 33-78 years (mean age $51 \pm 10.6$ ). Every case will be followed up for 5 years as part of their treatment following breast cancer diagnosis with a mean time of 21 months.

A total of 25 cases of Strattice ${ }^{\mathrm{TM}}$-based reconstruction had adjuvant radiotherapy. Of these, we report 8 cases (32\%) of complications requiring removal of implants. There were 4 cases $(16 \%)$ with poor skin compliance, where the skin failed to expand following inflation of expander; 2 cases $(8 \%)$ with wound break down after 4 and 9 months, respectively; 1 case (4\%) developed severe capsular contracture of the reconstructed breast and 1 case (4\%), where the implant ruptured. See Table 1 for a summary of complications.

The patients were further divided, and a breakdown of complications by groups and further procedures performed is shown in Table 2 . The majority (75\%) of complications occurred in group A.

\section{Chi-square analysis}

\begin{tabular}{llll}
\hline & No & Complications & Marginal row totals \\
& complications & & \\
Group A & $10(10.88)[0.07]$ & $6(5.12)[0.15]$ & 16 \\
Group B & $7(6.12)[0.13]$ & $2(2.88)[0.27]$ & 9 \\
$\begin{array}{c}\text { Marginal } \\
\text { column totals }\end{array}$ & 17 & 8 & 25 (grand total) \\
\hline
\end{tabular}

Table 2 Complications and further procedures

\begin{tabular}{|c|c|c|c|}
\hline & Complications & Number of patients $(N)$ & Further procedures \\
\hline \multirow[t]{3}{*}{ Group A } & Poor skin compliance & $N=4 / 16(25 \%)$ & Three LD-based reconstruction \\
\hline & & & One declined further surgery \\
\hline & Wound break down & $N=2 / 16(12.5 \%)$ & Implants removed and LD reconstructions \\
\hline \multirow[t]{2}{*}{ Group B } & Rupture of expander & $N=1 / 9(11.1 \%)$ & Replaced with new ones \\
\hline & Severe capsular contracture & $N=1 / 9(11.1 \%)$ & LD reconstruction \\
\hline
\end{tabular}

Chi-square analysis demonstrated independence of the timing of radiotherapy and the risk of developing complications $(p=0.43)$.

\section{Discussion}

Strattice $^{\mathrm{TM}}$ is non cross-linked acellular matrix which is derived from porcine dermis. Using this as a connective tissue graft material improves the quality of soft tissue in implantbased breast reconstruction [9]. Strattice ${ }^{\mathrm{TM}}$ incorporates into the recipient tissue with associated cells and microvascular ingrowths. It is completely an avascular tissue and gets vascularised from surrounding healthy tissue starting as early as around 2 weeks post-implantation and mature vascular structure at 6 months $[9,10]$.

It is known that stretching of tissue is associated with a significantly higher complication rate [11-15]. If radiation is given to this tissue, it is thought to add to further complications. Radiotherapy adversely affects hair, sweat and sebaceous gland function required for re-epithelialisation and wound repair and, in this way, compromises wound healing [3-5]. Some researchers believe the effect of radiation is similar to that of heavy smokers and discourage breast reconstruction in such cases [16]. However, there are studies which claim its protective effect, limiting chronic inflammation when exposed to radiation and subsequently reducing capsule formation [8]. In our group of patients, none of the smokers developed any complications but due to the small numbers, we are unable to suggest whether smoking is a risk factor or whether radiation provided any protection.

Park et al. had shown a high implant loss of $28 \%$ [6]. In a very recent study by Barber et al. [7], the reconstruction loss rate was quoted to be as high as $47.6 \%$. Current general opinion is that radiation is a risk for higher rate of ADM complications as such there is a bias in terms of amount of information available regarding this [17]. With the emergence of data suggesting the protective effect of ADM, it is necessary to identify the consequence of timing of radiation in ADM reconstruction [8]. Furthermore, Barber [7] also describes that most complications happen in those receiving pre-radiotherapy reconstruction. In the present study, the majority of patients had delayed reconstruction, having already 
completed their oncological treatment. Of those who had preradiotherapy reconstruction, there was only one patient who developed severe contracture but this did not delay treatment.

Overall significant complications were $32 \%$ in our study. Perhaps, surprisingly, there were no cases of infection noted in either group. In a study by Mitchel et al. [3], they attributed their low complication and infection to a thorough antibiotic irrigation of the subpectoral pocket, soaking of implant in antibiotic solution and adequate drainage of the cavity. We believe that among these, only adequate drainage of seroma had a major role to play in our study group to minimise infection. For this reason, we emphasise on suturing Strattice ${ }^{\mathrm{TM}}$ without folds, adequately filling the expander to close any dead space between it and mastectomy flap and removing the drain when there was minimal output.

The major limitation to this study is the relatively short duration of follow-up. It is entirely possible that in time, this population of patients could develop further wound problems or extrusion of implants. However, this is unpredictable as there is a paucity of data regarding the long-term outcomes in this population.

Although this present patient population is small and heterogenic, our results encourage us to continue to offer patients delayed and immediate reconstruction after thorough counselling and clear information provided to the patients of the high risk of reconstructive failure.

\section{Conclusion}

The evidence suggests that implant-based reconstruction using StratticeTM should not be contraindicated in pre or post-radiotherapy patients. However, when planning these procedures, it is paramount that the increased risks are emphasised to patients in order to better manage patient expectation in cases where complications arise.

Acknowledgments We would like to thank Lynn Pounder, a breast care specialist nurse, for her involvement in data collection and her continuous care for the patients in this article.

Ethical standards For this retrospective study formal consent from a local ethics committee is not required.

Funding There was no external funding for the study.

Conflict of interest Cho Ee Ng, Andrew Pieri and Tarannum Fasih declare that they have no conflict of interest.

Patient consent Patients provided written consent prior to their inclusion in the study.

\section{References}

1. Lardi AM, Ho-Asjoe M, Mohanna P-N, Farhadi J (2014) Immediate breast reconstruction with acellular dermal matrix: factors affecting outcome. J Plast Reconstr Aesthet Surg 67:10981105

2. Hirsch EM, Dumanian GA (2012) Discussion: AlloDerm and Strattice in breast reconstruction: a comparison and techniques for optimizing outcomes. Plast Reconstr Surg 129:1234-1235

3. Mitchell RE (2013) Porcine acellular dermis-assisted breast reconstruction: influence of adjuvant radiotherapy on complications and outcomes. Plast Reconstr Surg Glob open 1:e77

4. Bernstein EF, Sullivan FJ, Mitchell JB et al (1993) Biology of chronic radiation effect on tissues and wound healing. Clin Plast Surg 20:435-453

5. Burns JL, Mancoll JS, Phillips LG (2003) Impairments to wound healing. Clin Plast Surg 30:47-56

6. Parks JW, Hammond SE, Walsh WA et al (2012) Human acellular dermis versus no acellular dermis in tissue expansion breast reconstruction. Plast Reconstr Surg 130:739-746

7. Barber MD, Williams L, Anderson EDC et al (2015) Outcome of the use of acellular-dermal matrix to assist implant-based breast reconstruction in a single centre. Eur J Surg Oncol 41: $100-105$

8. Moyer HR, Pinell-White X, Losken A (2014) The effect of radiation on acellular dermal matrix and capsule formation in breast reconstruction: clinical outcomes and histologic analysis. Plast Reconstr Surg 133:214-221

9. Himsl I, Drinovac V, Lenhard M et al (2012) The use of porcine acellular dermal matrix in silicone implant-based breast reconstruction. Arch Gynecol Obstet 286:187-192

10. Connor J, McQuillan D, Sandor M et al (2009) Retention of structural and biochemical integrity in a biological mesh supports tissue remodeling in a primate abdominal wall model. Regen Med 4:185195

11. Hughes K, Brown C, Perez V et al (2012) The effect of radiotherapy on implant-based breast reconstruction in the setting of skin-sparing mastectomy: clinical series and review of complications. Anticancer Res 32:553-557

12. McCraw JB, Horton CE, Grossman JA et al (1987) An early appraisal of the methods of tissue expansion and the transverse rectus abdominis musculocutaneous flap in reconstruction of the breast following mastectomy. Ann Plast Surg 18:93-113

13. Kroll SS, Baldwin B (1992) A comparison of outcomes using three different methods of breast reconstruction. Plast Reconstr Surg 90: 455-462

14. Disa JJ, Cordeiro PG, Heerdt AH et al (2003) Skin-sparing mastectomy and immediate autologous tissue reconstruction after wholebreast irradiation. Plast Reconstr Surg 111:118-124

15. Tadiparthi S, Alrawi M, Collis N (2011) Two-stage delayed breast reconstruction with an expander and free abdominal tissue transfer: outcomes of 65 consecutive cases by a single surgeon. J Plast Reconstr Aesthet Surg 64:1608-1612

16. Nava MB, Cortinovis U, Ottolenghi J et al (2006) Skin-reducing mastectomy. Plast Reconstr Surg 118:603-610

17. Valdatta L, Cattaneo AG, Pellegatta I et al (2014) Acellular dermal matrices and radiotherapy in breast reconstruction: a systematic review and meta-analysis of the literature. Plast Surg Int 2014: 472604 